\title{
Preventive Effect of Oral Administration of Pantoea agglomerans-derived LPS in a Hypertensive Rat Model Upon Salt Loading
}

\author{
RAN ZHANG $^{1,2}$, HIROYUKI INAGAWA ${ }^{1,3}$, SACHIKO UCHIBORI $^{1}$, \\ TSUTOMU MASAKI ${ }^{4}$, HIDEKI KOBARA ${ }^{4}$ and GEN-ICHIRO SOMA ${ }^{1,3}$ \\ ${ }^{I} R \& D$ Division, Control of Innate Immunity, Technology Research Association, Kagawa, Japan; \\ ${ }^{2} R \& D$ Division, Umeken CO., LTD, Osaka, Japan; \\ ${ }^{3}$ Niigata University of Pharmacy and Applied Life Sciences, Niigata, Japan; \\ ${ }^{4}$ Department of Gastroenterology and Neurology, Faculty of Medicine, Kagawa University, Kagawa, Japan
}

\begin{abstract}
Background/Aim: Oral administration of Pantoea agglomerans-derived lipopolysaccharide (LPSp) has been reported to have a preventive effect against various lifestylerelated diseases. Therefore, we examined the preventive effect on high blood pressure, which is a kind of reserve arm for lifestyle-related diseases. Materials and Methods: Spontaneous hypertensive rat (SHR) and WKY rat were bred from 6 to 16 weeks of age. SHR were orally administered $100 \mu \mathrm{g} / \mathrm{kg} \mathrm{LPSp}$ and $0.1 \% \mathrm{NaCl}$, and blood pressure was measured at 6, 10,13 and 16 weeks. Furthermore, at 16 weeks of age, blood biochemical markers were measured and microbial community composition was analyzed. Results: SHRs developed hypertension with age, which was exacerbated by salt loading. Although there was almost no reduction in blood pressure in SHRs that received LPSp. It was suppressed at 13-16 weeks of age in those with salt loading. Conclusion: Oral administration of LPSp showed a preventive effect on salt-loaded hypertension.
\end{abstract}

Hypertension is the most prevalent disease globally. Patients with hypertension represent approximately $30 \%$ of the global population (1). In Japan, approximately 43 million people are estimated to have hypertension (2). In recent years, in addition to waist circumference (males $\geq 85 \mathrm{~cm}$ and females $\geq 90 \mathrm{~cm}$ ), systolic blood pressure (maximum value) $\geq 130 \mathrm{mmHg}$ and/or diastolic blood pressure (minimum value) $\geq 85 \mathrm{mmHg}$ have also been included in the diagnostic standards of metabolic

Correspondence to: Ran Zhang, Ph.D., Control of Innate Immunity, Technology Research Association, 2217-16 FROM-KAGAWA Bio Lab., Hayashi-cho, Takamatsu, Kagawa, 761-0301, Japan. Tel/Fax: +81878139203, e-mail: zhang@shizenmeneki.org

Key Words: Hypertensive, salt loading, Pantoea agglomeransderived LPS, oral administration. syndrome (3). Hypertension is considered as one indicator of metabolic syndrome. Hypertension is a risk factor for several diseases, such as vascular endothelial dysfunction, ischemic heart disease, stroke, and renal failure; the higher the blood pressure, the higher the risk of morbidity and mortality (4-7).

At present, the main treatments for hypertension include drug treatment with a diuretic agent, $\mathrm{Ca}$ antagonist, angiotensin-converting enzyme inhibitor, angiotensin II receptor antagonist, or a sympathetic agent. However, sideeffects are common in drug therapy; therefore, it is desirable to improve lifestyle habits prior to drug therapy, unless urgent treatment is required. Particularly for normotensives and mild hypertensives, non-drug dependent prevention is considered important.

Hypertension is a complex trait determined by both genetic and environmental factors and is a major public health problem due to its high prevalence and concomitant increase in the risk of cardiovascular disease (8). Among environmental factors associated with hypertension, dietary salt intake is the most common and important risk factor (9). In addition, the alleviation of hypertension due to high salt intake is related to the production of vascular endothelial growth factor c (VEGFc) by macrophages and their removal of salt from the body through lymphatic vessels. Conversely, macrophage hypofunction leads to hypertension upon high salt intake (10).

In this study, lipopolysaccharide (LPS) derived from the plant symbiotic gram-negative bacteria Pantoea agglomerans (LPSp), and its preventive effects on various diseases were examined (11-14). LPS is a component of the outer cell membrane of gram-negative bacteria and comprises lipids and polysaccharides. The physiological action of LPS is exerted through Toll-like receptor 4 present on the cell surface of host cells (14). LPSp is produced by a type of gram-negative bacteria which supply nitrogen and phosphorus to a wide 
range of plants during symbiosis (15-16). Moreover, in animals, oral administration of LPSp improves lifestyle-related diseases, including hyperlipidemia in rabbits, diabetes in obese mice, and cognitive function in mice with Alzheimer's disease, and suppresses arteriosclerotic lesions in mice (17-20). It is thus, recognized to have various preventive effects in different diseases. The oral administration of LPSp also improves parameters related to glucose and lipid metabolism. The efficacy of oral administration of LPSp may involve macrophage activation (21). Hypertension due to high salt intake may be improved by macrophage activation. In this study, the antihypertensive effect of oral administration of LPSp was examined in a spontaneously hypertensive rat model received high salt.

\section{Materials and Methods}

Animals and treatments. Male 6-week-old SHR/NCrlCrlj (SHR) and WKY/NCrlCrlj (WKY) were obtained from Charles River Laboratories Japan (Yokohama, Japan). Care and handling of animals were in accordance with the Guidelines for the Care and Use of Laboratory Animals at Kagawa University and were approved by Kagawa University Institutional Animal Care and Use Committee. Rats were housed under conditions of controlled temperature and humidity with a 12-h light/dark cycle and unrestricted access to food and water. Low-fat diet (LFD; $16.1 \mathrm{~kJ} / \mathrm{g}$, $10 \%$ of energy as fat, D12450B) was purchased from Research Diets, Inc. (New Brunswick, NJ, USA). After 1 week of prefeeding, rats were divided into the following groups ( $n=2-4$ per group): WKY-DW group, SHR (DW) group, SHR-LPSp (LPS) group, SHR$0.1 \% \mathrm{NaCl}(\mathrm{Na})$ group, and SHR- $0.1 \% \mathrm{NaCl}+\mathrm{LPSp}$ (Na+LPS) group. Purified LPS derived from P. agglomerans (LPSp; Macrophi Inc., Kagawa, Japan) was dissolved in drinking water and applied at $0.1 \mathrm{mg} / \mathrm{kg}$ body weight/day (21-22). $\mathrm{NaCl}$ was adjusted to $0.1 \%$ with tap water (9). Each rat was then fed a low-fat diet for 10 weeks. Systolic blood pressure (SBP), diastolic blood pressure (DBP), and mean blood pressure (MBP) were monitored using tailcuff methods (BP-98A; Softron Co., Tokyo, Japan) at 6, 10, 13, and 16 weeks of age. At 16 weeks of age, blood was collected from the heart of these rats, and plasma was obtained by centrifugation. All surgeries were performed under diethyl ether anesthesia, and rats were euthanized via diethyl ether inhalation.

Measurement of biochemical markers. Levels of plasma biochemical markers [triglyceride (TG), total cholesterol (T-CHO), low-density-lipoprotein (LDL-C) and high-density-lipoprotein (HDL-C) cholesterol, aspartate transaminase (AST), alanine aminotransferase (ALT), and total-bilirubin (T-BIL) and oxidized LDL/LDL (OxLDL/LDL)] were commissioned to Oriental Yeast Co. Ltd (Japan).

Microbial community analysis. Microbial DNA was extracted from freeze-dried rat fecal samples using the MPure Bacterial DNA Extraction Kit (Qiagen, Hilden, Germany). Amplification, sequencing, and analysis of extracted DNA were commissioned to Bioengineering Lab (Chiba, Japan). Sequencing was performed using MiSeq under conditions of $2 \times 300 \mathrm{bp}$. Analysis of alpha and beta diversity was performed using QIIME script. The database was
97\% OTU of Greengene attached to the bacterial flora analysis pipeline QIIME, and all sequences not judged to be chimeras were extracted and used for the subsequent analysis.

Statistical analysis. All results are expressed as mean \pm SEM. If a difference based on one- or two-way analysis of variance was significant, then Bonferroni's multiple comparison test was used for paired comparisons using GraphPad Prism for Windows (GraphPad Software, Inc., San Diego, CA, USA). Results were considered significant at $p<0.05$.

\section{Results}

Changes in blood pressure over time between WKY-DW group, DW group and LPS group. Compared with the WKYDW group, SBP, DBP and MBP in the DW group and LPS group were significantly increased, but no significant difference was found between the DW group and LPS group (Figure 1).

Changes in blood pressure over time between WKY-DW group, Na group and Na+ LPS group. Compared with WKYDW group, SBP, DBP and MBP of Na group and Na+LPS group increased significantly. In DBP, there was a significant difference between DW group and Na+LPS group, but in SBP and MBP, there was no significant difference between DW group and LPS group. In addition, SBP, DBP and MBP in the Na+LPS group decreased compared to the Na group, but no significant difference was observed (SBP: $p=0.0587$, DBP: $p=0.1421$, MBP: $p=0.1546$, respectively) (Figure 2).

Analysis of biochemical markers between DW group, LPS group, Na group and Na+LPS group. Several biochemical markers were evaluated in the DW group, LPS group, Na group, and Na+LPS group. TP, ALT, GLU, and AMY, did not differ significantly between the four groups. Regarding TG, the LPS group and $\mathrm{Na}$ group showed significant decreases compared with the DW group. Regarding AST, the Na+LPS group showed a decrease, whereas the $\mathrm{Na}$ group showed an increase, compared with the LPS group, but these differences were not significant. Regarding HDL$\mathrm{C}$, the Na+LPS group showed a tendency to increase compared with the $\mathrm{Na}$ group, but this difference also was not significant (Figure 3).

Gut microbiota community analysis. Gut microbiota composition was analyzed by extracting microbial DNA from stool samples. As shown in Figure 4A, relative abundance of bacteria was calculated at the phylum level. There was no difference in this regard between the DW group and LPS group, but the Na group showed an increase in the abundance compared with these two groups. Compared with the Na group, the Na+LPS group showed a decrease in the relative abundance of Firmicutes/ 


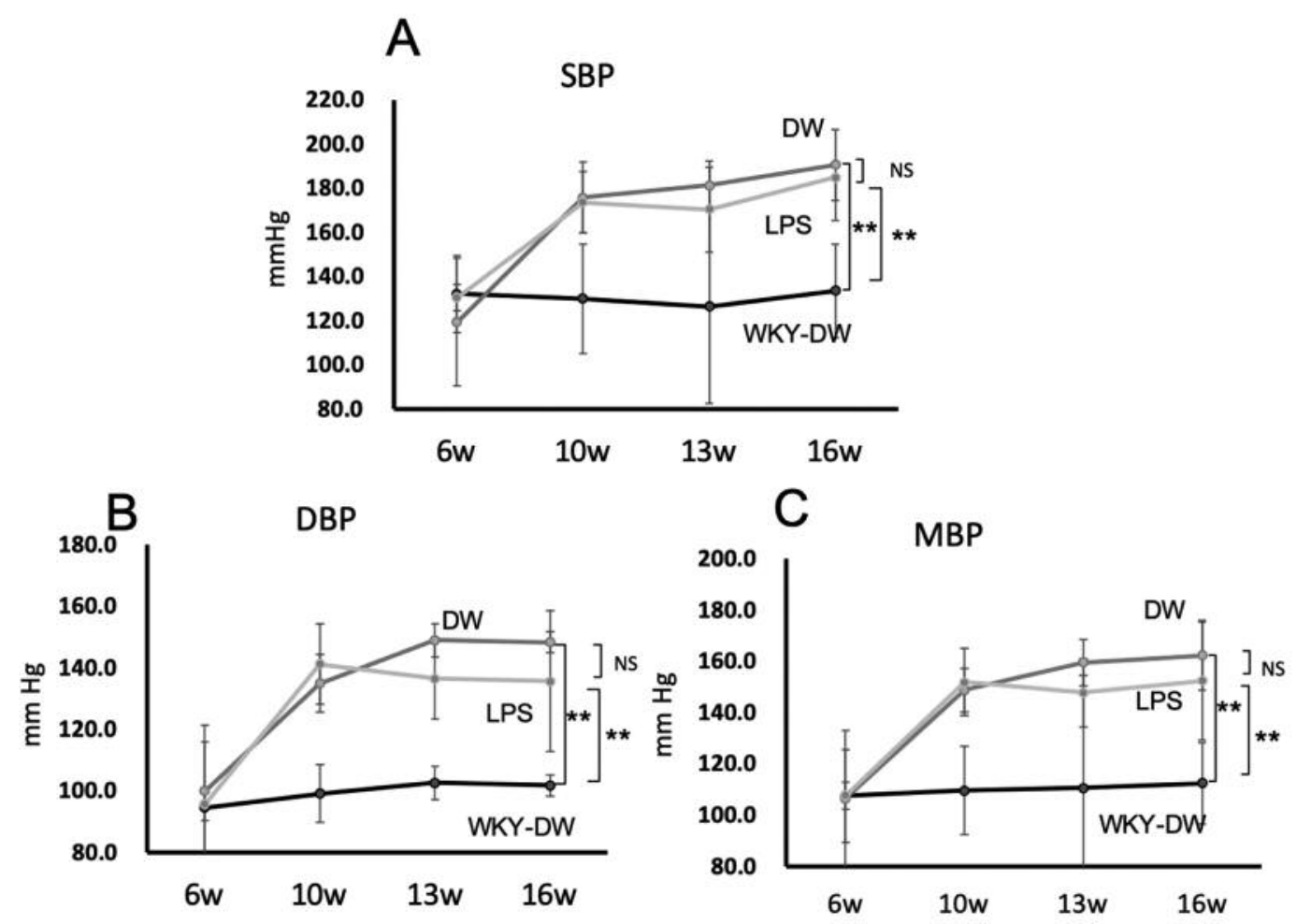

Figure 1. Changes in blood pressure over time in the WKY-DW group, DW group and LPS group. SBP (A), DBP (B) and MBP (C) were measured at 6 weeks old, 10 weeks old, 13 weeks old, 16 weeks old rats. Black: WKY-DW group, gray: DW group, light gray: LPS group. The data are presented as mean $\pm S E M$ and were obtained from $2-4$ rats per group. ${ }^{*} p<0.05,{ }^{*} p<0.01, * * * p<0.001$ compared to the WKY-DW group.

Bacteroidetes; however, the difference was not significant (Figure 4B). Furthermore, weighted UniFrac analyses were used to calculate distances between the fecal samples from the DW group and LPS group (Figure 4C), Na group and $\mathrm{Na}+\mathrm{LPS}$ group (Figure 4D). 3-dimensional (3D) scatterplots were generated by principal coordinate analysis (PCoA) to visualize whether the experimental groups in the input phylogenetic tree have significantly different microbial communities. This method allows us to present dissimilarities of the data in terms of distance. The composition of the fecal microbial communities of the DW group and LPS Group were not found to be distinct (Figure 4C). However, the $\mathrm{Na}$ group and $\mathrm{Na}+\mathrm{LPS}$ group were found to be distinct (Figure 4D). A clear separation was observed in the PCoA between the 2 clusters, representing the microbial compositions of the $\mathrm{Na}$ group and $\mathrm{Na}+\mathrm{LPS}$ group, indicating 2 extremely different gut environments.

\section{Discussion}

Epidemiological studies have shown that high blood pressure is a risk factor for stroke and cardiovascular disease, diabetes, aging, and dyslipidemia. The commonly used SHR hypertensive model rat also has abnormal lipid metabolism as well as spontaneous hypertension (4-7). In this study, although SBP, MBP, and DBP increased over time, oral administration of LPSp at $0.1 \mathrm{mg} / \mathrm{kg}$, improved slightly blood pressure (Figure 1). However, when the Na group was administered $0.1 \mathrm{mg} / \mathrm{kg}$ LPSp, there was a suppression of the increase in blood pressure compared with that in the nontreated $\mathrm{Na}$ group (Figure 2).

High salt intake is associated with high blood pressure and increased risk of cardiovascular disease (9). Numerous basic and clinical studies have shown that excessive salt intake can increase blood pressure. Salt intake acts not only on hemodynamics but also on macrophages, which play a role in regulating intradermal sodium concentration (10).

When salt intake exceeds the ability of salt excretion in the kidney, it mainly accumulates subcutaneously. This subcutaneously accumulated sodium is then excreted from the lymphatic vessels in a manner controlled by macrophages via VEGFc production (10). As such, if the function of macrophages decreases, then it may lead to high blood pressure. In this study, oral administration of LPSp was effective at preventing hypertension upon salt intake. It is hypothesized that LPSp acting on maintenance and enhancement of macrophage function, promotes elimination of salt to suppress hypertension. 


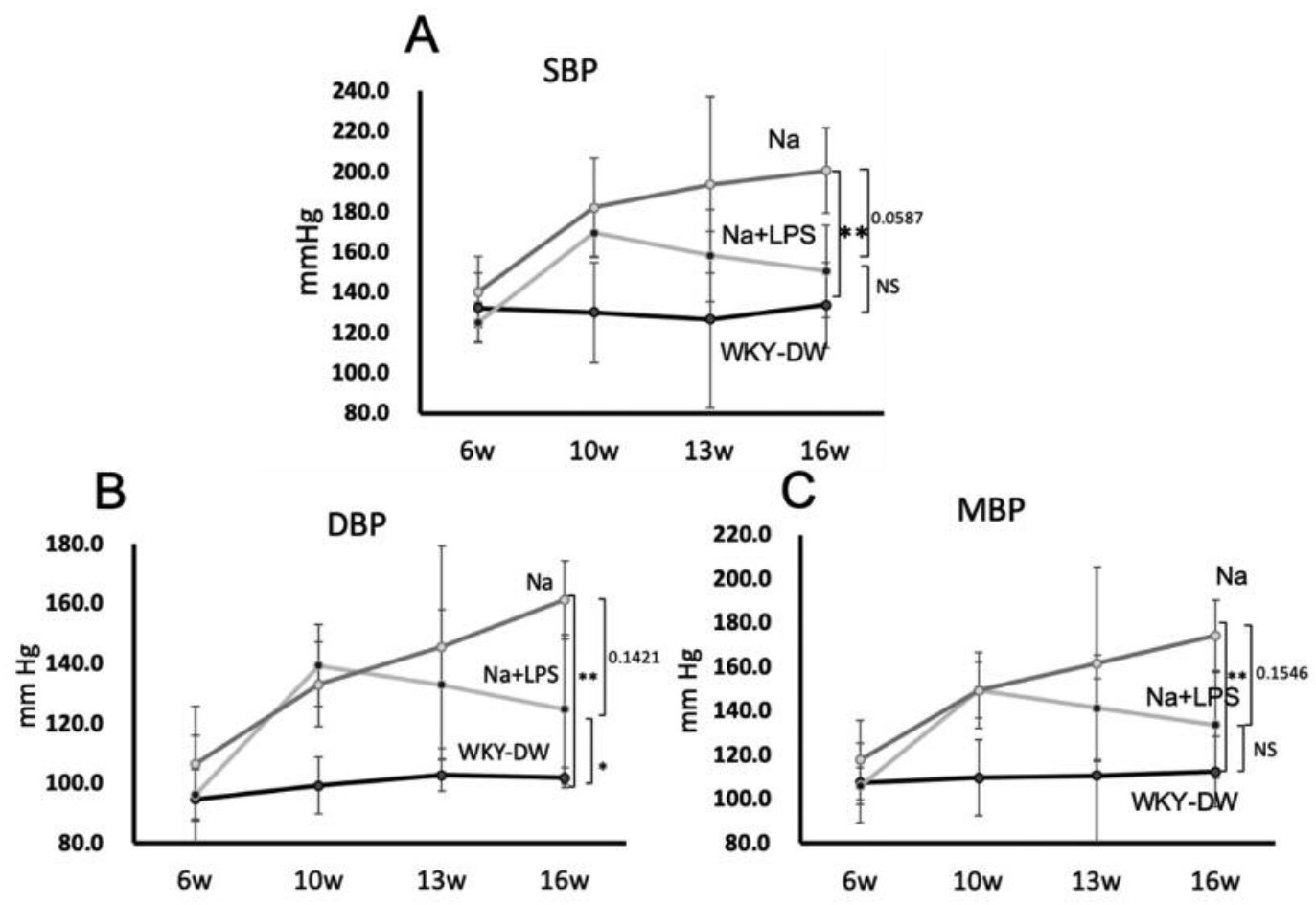

Figure 2. Changes in blood pressure over time in the WKY-DW group, Na group and Na+LPS group. SBP $(A), D B P(B)$ and $M B P(C)$ were measure at 6 weeks old, 10 weeks old, 13 weeks old, 16 weeks old rats. Black: WKY-DW group, gray: Na group, light gray: Na+LPS group. The data are presented as mean $\pm S E M$ and were obtained from $2-4$ rats per group. ${ }^{*} p<0.05, * * p<0.01, * * * p<0.001$ compared to the WKY-DW group.
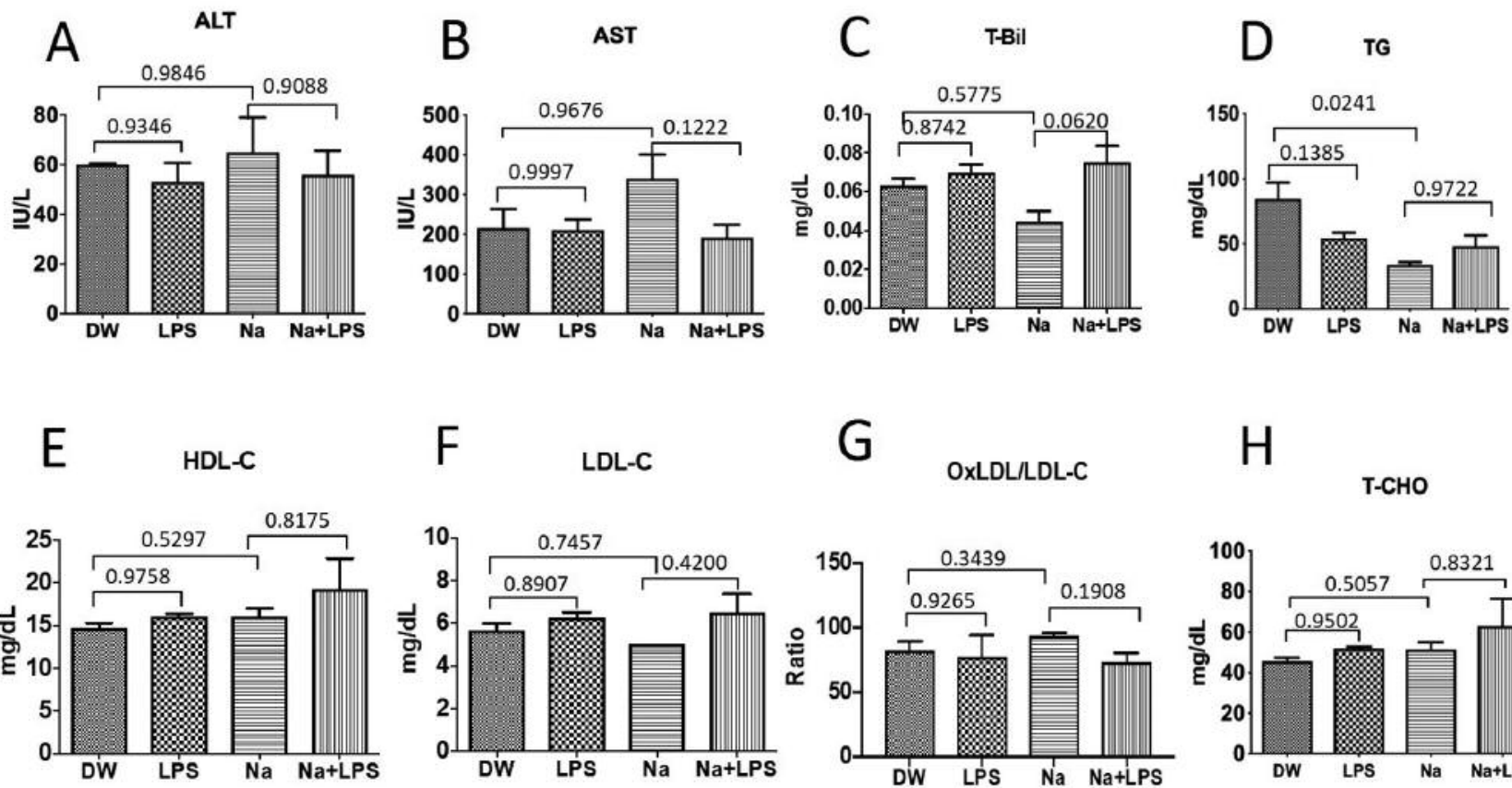
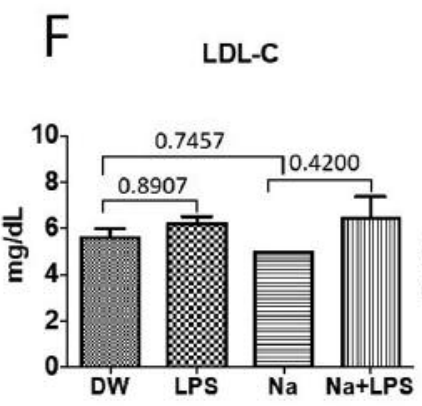

OxLDL/LDL-C

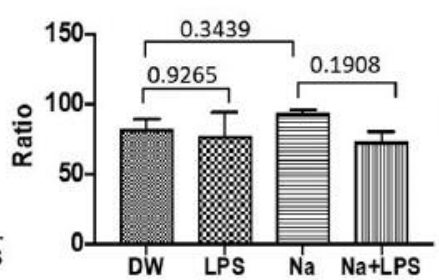

$\mathrm{H}$

T-CHO

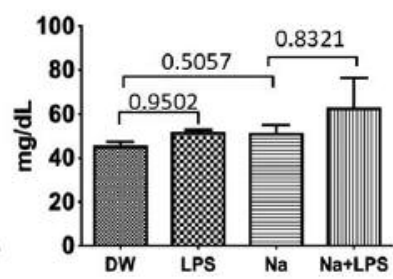

Figure 3. The levels of plasma biochemical markers in the DW group, LPS group, Na group and Na+LPS group. ALT: alanine aminotransferase (A), AST: Aspartate transaminase (B), T-BIL: total bilirubin (C), TG: triacylglycerol (D), HDL-C: high density lipoprotein cholesterol (E), LDL-C: low density lipoprotein cholesterol $(F), O x L D L / L D L$ : oxidized $L D L / L D L(G), T-C H O$ : total cholesterol $(H)$. The data are presented as mean $\pm S E M$ and were obtained from 2-4 rats per group. 

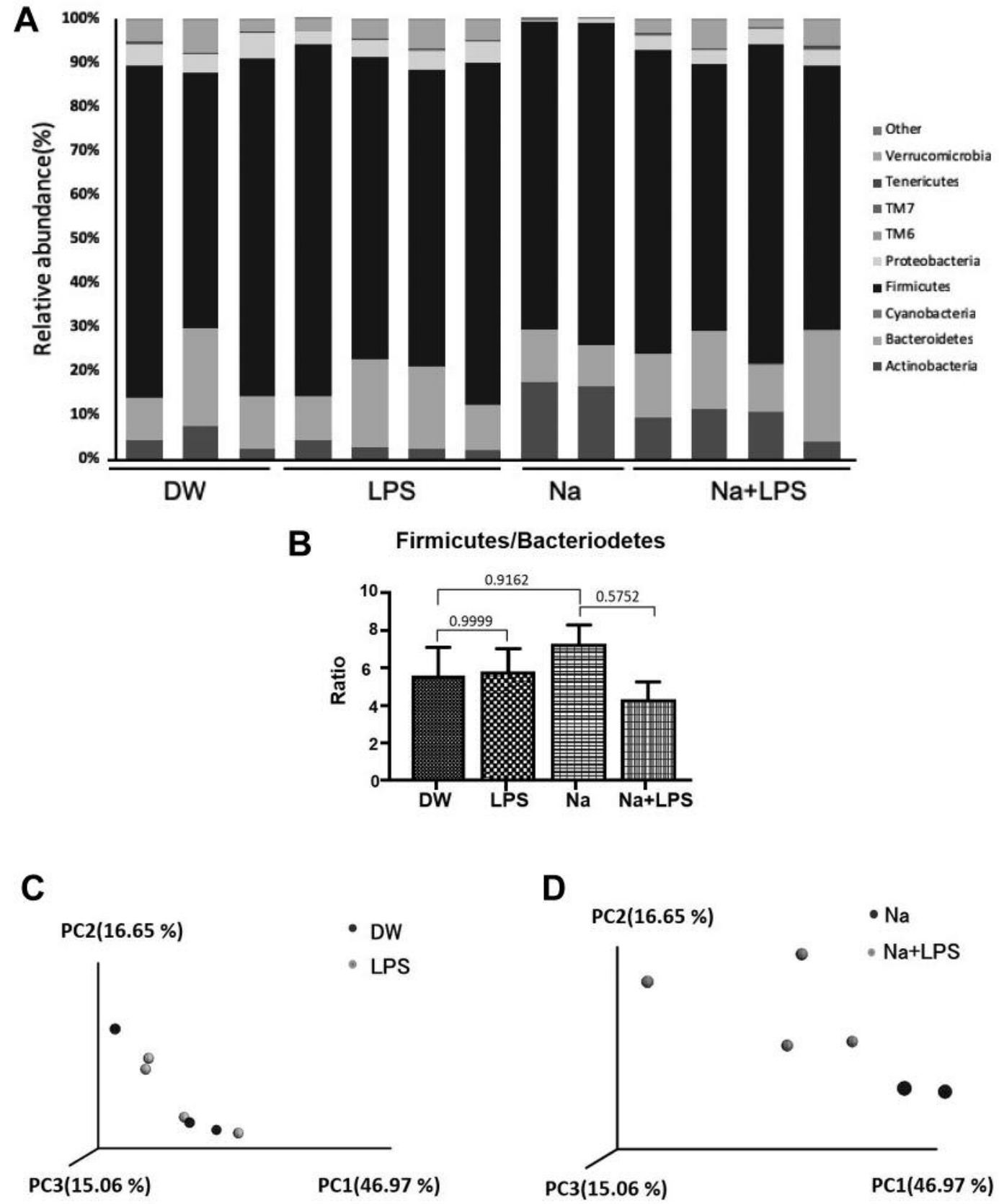

Figure 4. Gut microbiota composition in the DW group, LPS group, Na group and Na+LPS group. Variations in bacterial community compositions in stool samples at the phylum levels. The ratio of Bacteroidetes to Firmicutes (B). Principal component analysis results from stool samples of DW and LPS group (C). C: Black circle: DW group; Gray circle: LPS group. Principal component analysis results from stool samples of Na and Na+LPS group (D). D: Black circle: Na group; Gray circle: Na+LPS group. The data were obtained from 2-4 rats per group.

In recent years, many studies related to intestinal microflora and disease onset have been reported (22). According to the results of a study on intestinal flora and obesity, the intestinal bacterial composition differs between obese and normal mice kept in the same environment. In obese mice, the abundance of Firmicutes is increased and that of Bacteroidetes is decreased; thus, the ratio of
Firmicutes/Bacteroidetes ( $\mathrm{F} / \mathrm{B}$ ) is increased (22). In addition, in a hypertensive rat model, the $\mathrm{F} / \mathrm{B}$ ratio has been reported to be increased as in obesity, and the diversity of intestinal bacterial flora is decreased (23). However, LPSp intake brought the F/B ratio to the same level as in DW group. The level of Bacteroidetes was decreased by $\mathrm{NaCl}$, but it was normalized by oral administration of LPSp (Figure 4B). Oral 
administration of LPSp to ApoE-deficient mice in an arteriosclerosis model resulted in a significant decrease in enteric flora compared to that in the control group, along with an increase in the level of Bacteroidetes (19). This study produced similar results.

Hypertension is mainly caused by obesity and kidney injury. However, in contrast to DW group and $\mathrm{Na}$ group, the administration of LPSp did not result in significant changes in biochemical markers, such as lipid and liver metabolism levels (Figure 3). Although the mechanism of improvement of salt intake-induced hypertension by LPSp is unclear, macrophages may be involved in promoting sodium excretion. Based on the above data, we plan to analyze the role of macrophages in salt excretion.

\section{Conflicts of Interest}

The Authors have no financial conflicts of interest.

\section{Authors' Contributions}

R. Z. and H. I. were responsible for the study concept and design; R. Z. and S.U. was responsible for acquisition of data; T. M. and H. $\mathrm{K}$. were responsible for analysis and interpretation of data; R. Z. was responsible for drafting the manuscript; and G. S. was responsible for study supervision.

\section{Acknowledgements}

This work was partly supported by the Council for Science, Technology and Innovation (CSTI), Cross-ministerial Strategic Innovation Promotion Program (SIP), "Technologies for creating nextgeneration agriculture, forestry and fisheries" (funding agency: Biooriented Technology Research Advancement Institution, NARO).

\section{References}

1 Wolf-Maier K, Cooper RS, Banegas JR, Giampaoli S, Hense HW, Joffres M, Kastarinen M, Poulter N, Primatesta P, Rodríguez-Artalejo F, Stegmayr B, Thamm M, Tuomilehto J, Vanuzzo D and Vescio F: Hypertension prevalence and blood pressure in 6 European countries, Canada, and the United States. JAMA 289(18): 2363-2369, 2003. PMID: 12746359. DOI: 10.1001/jama.289.18.2363

2 Ikeda N, Saito E, Kondo N, Inoue M, Ikeda S, Satoh T, Wada K, Stickley A, Katanoda K, Mizoue T, Noda M, Iso H, Fujino Y, Sobue T, Tsugane S, Naghavi M, Ezzati M and Shibuya K: What has made the population of Japan healthy? Lancet 378(9796): 1094-1105, 2011. PMID: 21885105. DOI: 10.1016/ S0140-6736(11)61055-6

3 Miura K, Nagai M and Ohkubo T: Epidemiology of hypertension in Japan. Circ J 77: 2226-2231, 2013. PMID: 23902998. DOI: https://doi.org/10.1253/circj.CJ-13-0847

4 He J, Ogden LG, Vupputuri S, Bazzano LA, Loria C and Whelton PK: Dietary sodium intake and subsequent risk of cardiovascular disease in overweight adults. JAMA 282: 20272034, 1999. PMID: 10591385. DOI:10.1001/jama.282.21.2027
5 Tuomilehto J, Jousilahti P, Rastenyte D, Moltchanov V, Tanskanen A, Pietinen $\mathrm{P}$ and Nissinen A: Urinary sodium excretion and cardiovascular mortality in Finland: a prospective study. Lancet 357: 848-851, 2001. PMID: 11265954. DOI: 10.1016/S0140-6736(00)04199-4

6 Nancy RC, Eva O, Julie EB, Kathryn MR, Shiriki KK, Lawrence JA and Paul KW: Long term effects of dietary sodium reduction on cardiovascular dis- ease outcomes: observational follow up of the trials of hypertension prevention (TOHP). BMJ 334: 885-888, 2007. PMID: 17449506. DOI: 10.1136/ bmj.39147.604896.55

7 Strazzullo P, D'Elia L, Kandala NB and Cappuccio FP: Salt intake, stroke, and cardiovascular disease: meta-analysis of prospective studies. BMJ 339: b4567-b4576, 2009. PMID: 19934192. DOI: $10.1136 /$ bmj.b4567

8 Metabolic syndrome Drome diagnostic criteria Review Committee: Metabolic syndrome definition and diagnostic criteria. J Jpn Soc Int Med 94(4): 188-203, 2005. PMID: 16394611. DOI: $10.5551 /$ jat.12.301

9 Machnik A, Dahlmann A, Kopp C, Goss J, Wagner H, van Rooijen N, Eckardt KU, Müller DN, Park JK, Luft FC, Kerjaschki $\mathrm{D}$ and Titze $\mathrm{J}$ : mononuclear phagocyte system depletion blocks interstitial tonicity-responsive enhancer binding protein/vascular endothelial growth factor $\mathrm{c}$ expression and induces salt-sensitive hypertension in rats. Hypertension 55(3): 755-761, 2010. PMID: 20142563. DOI: 10.1161/HYPERTENSIONAHA.109.143339

10 Machnik A, Neuhofer W, Jantsch J, Dahlmann A, Tammela T, Machura K, Park JK, Beck FX, Müller DN, Derer W, Goss J, Ziomber A, Dietsch P, Wagner H, van Rooijen N, Kurtz A, Hilgers KF, Alitalo K, Eckardt KU, Luft FC, Kerjaschki D and Titze J: Macrophages regulate salt-dependent volume and blood pressure by a vascular endothelial growth factor-C-dependent buffering mechanism. Nat Med 15(5): 545-552, 2009. PMID: 19412173. DOI: 10.1038/nm.1960

11 Tamura Y, Inagawa H, Nakata Y, Kohchi C and Soma GI: Effects of the subaleurone layer of rice on macrophage activation and protection of pollen allergy in a murine model. Anticancer Res 35(8): 4467-4472, 2015. PMID: 26168488.

12 Amano S, Inagawa H, Nakata Y, Ohmori M, Kohchi C and Soma G: Oral administration of lipopolysaccharide of acetic acid bacteria protects pollen allergy in a murine model. Anticancer Res 35(8): 4509-4514, 2015. PMID: 26168494.

13 Komori T, Saito K, Sawa N, Shibasaki Y, Kohchi C, Soma GI and Inagawa $\mathrm{H}$ : Innate immunity activated by oral administration of LPSp is phylogenetically preserved and developed in broiler chickens. Anticancer Res 35(8): 4461-4466, 2015. PMID: 26168487.

14 Poltorak A, He X, Smirnova I, Liu MY, Van Huffel C, Du X, Birdwell D, Alejos E, Silva M, Galanos C, Freudenberg M, Ricciardi-Castagnoli P, Layton B and Beutler B: Defective LPS signaling in $\mathrm{C} 3 \mathrm{H} / \mathrm{HeJ}$ and $\mathrm{C} 57 \mathrm{BL} / 10 \mathrm{ScCr}$ mice: mutation in TLR4 gene. Science 282(5396): 2085-2088, 1998. PMID: 9851930. DOI: $10.1126 /$ science.282.5396.2085

15 Lindh E, Kjaeldgaard P, Frederiksen W and Ursing J: Phenotypical properties of Enterobacter agglomerans (Pantoea agglomerans) from human, animal and plant sources. APMIS 99(4): 347-352, 1991. PMID: 2036218.

16 Nunes C, Usall J, Teixido N and Vinas I: Biological control of postharvest pear diseases using a bacterium, Pantoea agglomerans CPA-2. Int J Food Microbiol 70(12): 53-61, 2001. PMID: 29856882. DOI: 10.1371/journal.pone.0198493 
17 Kobayashi Y, Inagawa H, Kohchi C, Kazumura K, Tsuchiya H, Miwa T, Okazaki K and Soma GI: Oral administration of Pantoea agglomerans-derived lipopolysaccharide prevents metabolic dysfunction and Alzheimer's disease-related memory loss in senescence-accelerated prone 8 (SAMP8) mice fed a high-fat diet. PLoS One 13(6): e0198493. 2018. PMID: 29856882. DOI: 10.1371/journal.pone.0198493

18 Okutomi T, Nishizawa T, Inagawa H, Takano T, Morikawa A, Soma G and Mizuno D: Homeostasis as regulated by activated macrophage. VII. Suppression of serum cholesterol level by LPSw (a lipopolysaccharide from wheat flour) in WHHL (Watanabe Heritable Hyperlipidemic) rabbit. Chem Pharm Bull 40: 1268-1270, 1992. PMID: 29584779. DOI: 10.1371/journal. pone. 0195008

19 Kobayashi Y, Inagawa H, Kohchi C, Kazumura K, Tsuchiya H, Miwa T, Okazaki K and Soma GI: Oral administration of Pantoea agglomerans-derived lipopolysaccharide prevents development of atherosclerosis in high-fat diet-fed apoE-deficient mice via ameliorating hyperlipidemia, pro-inflammatory mediators and oxidative responses. PLoS One 13(3): e0195008, 2018. PMID: 29584779. DOI: 10.1371/journal. pone. 0195008

20 Wakame $\mathrm{K}$, Komatsu $\mathrm{K}$, Inagawa $\mathrm{H}$ and Nishizawa $\mathrm{T}$ : Immunopotentiator from Pantoea agglomerans prevents atopic dermatitis induced by dermatophagoides farinae extract in $\mathrm{NC} / \mathrm{Nga}$ mouse. Anticancer Res 35(8): 4501-4508, 2015. PMID: 26168493.
21 Inagawa H, Kobayashi Y, Kohchi C, Zhang R, Shibasaki Y and Soma GI: Primed activation of macrophages by oral administration of lipopolysaccharide derived from Pantoea agglomerans. In Vivo 30(3): 205-211, 2016. PMID: 27107076.

22 Yang T, Santisteban MM, Rodriguez V, Li E, Ahmari N, Carvajal JM, Zadeh M, Gong M, Qi Y, Zubcevic J, Sahay B, Pepine CJ, Raizada MK and Mohamadzadeh M: Gut dysbiosis is linked to hypertension. Hypertension 65: 1331-1340, 2015. PMID: 25870193. DOI: 10.1161/HYPERTENSIONAHA.115.05315

23 Turnbaugh PJ, Ley RE, Mahowald MA, Magrini V, Mardis ER and Gordon JI: An obesity-associated gut microbiome with increased capacity for energy harvest. Nature 444: 1027-1031, 2006. PMID: 17183312 . DOI: $10.1038 /$ nature05414
Received May 23, 2019

Revised June 26, 2019

Accepted July 2, 2019 